\title{
Transitions from partial to complete generalized synchronizations in bidirectionally coupled chaotic oscillators
}

\author{
Zhigang Zheng, ${ }^{1,2}{ }^{*}$ Xingang Wang, ${ }^{1}$ and Michael C. Cross ${ }^{2}$ \\ ${ }^{1}$ Department of Physics, Beijing Normal University, Beijing 100875, China \\ ${ }^{2}$ Department of Physics, California Institute of Technology, Pasadena, California 91125 \\ (Received 10 December 2001; revised manuscript received 29 January 2002; published 8 May 2002)
}

\begin{abstract}
Generalized synchronization in an array of mutually (bidirectionally) coupled nonidentical chaotic oscillators is studied. Coupled Lorenz oscillators and coupled Lorenz-Rossler oscillators are adopted as our working models. With increasing the coupling strengths, the system experiences a cascade of transitions from the partial to the global generalized synchronizations, i.e., different oscillators are gradually entrained through a clustering process. This scenario of transitions reveals an intrinsic self-organized order in groups of interacting units, which generalizes the idea of generalized synchronizations in drive-response systems.
\end{abstract}

DOI: 10.1103/PhysRevE.65.056211

PACS number(s): 05.45.Xt, 05.45.Pq

Recently synchronization behaviors in coupled or driven chaotic elements have been extensively exploited both theoretically and experimentally in the context of many specific problems such as laser dynamics [1], electronic circuits [2], chemical and biological systems [3], and secure communications [4], due to its theoretical importance and application perspectives. The entrainment of coupled or driven limit cycles (periodic oscillators) has long been a widely studied topic, while the synchronization of chaotic oscillators was an open area due to the presence of the intrinsic nonlinearity [5]. People had thought synchronization of coupled chaotic oscillators cannot be attained because chaotic systems exhibit the exponential instability of nearby orbits (the socalled butterfly effect). However, this has been changed since it was shown by Pecora and Carroll and others [6] that two interacting identical chaotic oscillators can achieve synchronization [the complete or identical synchronization (CS)] i.e., they evolve on the synchronized manifold, $\mathbf{X}_{1}(t)=\mathbf{X}_{2}(t)$ $=\mathbf{X}(t)$, even though they individually possess the exponential instability of neighboring orbits. Due to the complicated feature of chaotic motion, there should be different levels of synchronized order. This study arouse extensive interest in synchronized entrainment of chaotic oscillators, and different degrees of synchronizations were found. CS appears only when interacting systems are identical. For two different chaotic oscillators it was found in 1995 for the drive-response systems that although CS can never be attained, the so-called generalized synchronization (GS) could be achieved, i.e., an emergence of some functional relation between the states of response and drive, i.e., $\mathbf{X}_{2}(t)=\mathbf{G}\left[\mathbf{X}_{1}(t)\right]$, can be observed [7]. In 1996 Rosenblum and co-workers observed the entrainment of phases for two coupled chaotic oscillators with small parameter mismatches, if an appropriate phase variable can be defined. This locking of phases admits the chaoticity and uncorrelation of oscillation amplitudes for the two chaotic oscillators. They call this locking behavior the phase synchronization (PS) [8]. The emergence of PS indicates an order in some degrees of freedom in coupled oscillator sys-

*Email address: zgzheng@bnu.edu.cn tems. Later on, the lag synchronization (LS) was found [9] for stronger coupling strengths, i.e., the two states are identified by a temporal shift, $\mathbf{X}_{2}(t)=\mathbf{X}_{1}(t+\tau)$. A weaker form of synchronization, i.e., the so-called measure synchronization was also proposed for Hamiltonian systems [10]. These explorations reveal rich levels of intrinsic orders in complicated nonlinear dynamics, which is absent in coupled limit cycles. Relation among these different levels of synchronization is subtle and received much attention recently.

For a group of interacting chaotic units most of the abovementioned forms of synchronizations exhibit some cascades from disorder to partial and global order when varying the coupling. For example, for coupled identical chaotic oscillators it was found that there is a cascade from partial synchronization to a CS with increasing the coupling strength [11]. The CS state may also lose its stability through a Hopf bifurcation to a traveling-wave state $[12,13]$. For PS, it has been well exhibited, by using both coupled limit cycles $[14,15]$ and coupled chaotic oscillators $[16,17]$, that a cascade of transitions from the partial PS to a global PS take place when one increases the coupling strength, and these transitions are accompanied by a number of topological changes in the phase space (i.e., the negativeness of one zero Lyapunov exponent at the threshold of PS, indicating the decrease of the topological dimension of the phase space by 1). LS is also expected to have a similar cascade for coupled chaotic oscillators [9]. Therefore, it may be a scenario that an internal cascade can be found for different kinds of synchronizations. An exception that still remains open is the generalized synchronization. Up to now, studies on the GS are rather limited. All the previous studies of GS have been concentrated on the drive-response system, which calls for the stability of the functional manifold $\mathbf{X}_{2}(t)=\mathbf{G}\left[\mathbf{X}_{1}(t)\right]$. To our knowledge, no studies on the GS for bidirectionally coupled oscillator systems have been found to date. For mutually (bidirectionally) coupled chaotic systems, no good way has been proposed in analyzing how the units achieve GS. Because oscillators are coupled to each other, it is important to study how these oscillators are self-organized and follow each other. Therefore, a number of questions can be proposed, for example: How can one say the units achieve GS? Is the GS for the two coupling directions achieved at the 


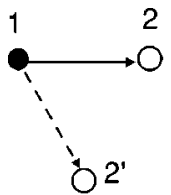

(a)

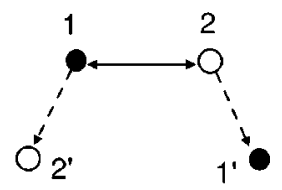

(b)
FIG. 1. (a) The auxiliary-element approach to detecting the generalized synchronization in the drive-response systems; (b) A schemetic approach of the auxiliary method to studying the generalized synchronization in two mutually coupled chaotic oscillators.

same coupling parameter or at different couplings? How can one determine the functional relationship between two chaotic units? and so on. In this paper we shall try to deal with the first two issues, which should be fundamental and treated as a first step to explore the GS behavior of the system with mutually interacting units.

In traditional studies on the generalized synchronization of drive-response systems, a simple way called auxiliary system approach has been proposed and widely used [18]. Consider the following drive-response system:

$$
\begin{gathered}
\dot{\mathbf{X}}_{1}=\mathbf{F}_{1}\left(\mathbf{P}_{1}, \mathbf{X}_{1}\right), \\
\dot{\mathbf{X}}_{2}=\mathbf{F}_{2}\left(\mathbf{P}_{2}, \mathbf{X}_{2}\right)+\varepsilon\left(\mathbf{X}_{1}-\mathbf{X}_{2}\right),
\end{gathered}
$$

where $\mathbf{X}_{1,2}(t)$ represent the dynamical flows in the phase space, $\mathbf{F}_{1,2}$ nonlinear function vectors with $\mathbf{P}_{1,2}$ the parameter sets for systems 1 (drive) and 2 (response) (usually different). $\varepsilon$ is the strength of the drive. Due to the nonlinearity, the flow of individual oscillators in phase space is usually chaotic. Usually 1 and 2 possess different chaotic flows. Let us consider an auxiliary system $2^{\prime}$ that is identical to the response system 2 (in the sense that they have the same equations of motions and parameters), as shown in Fig. 1(a). The equation of motion of the auxiliary system has the same form as that of 2

$$
\dot{\mathbf{X}}_{2}^{\prime}=\mathbf{F}_{2}\left(\mathbf{P}_{2}, \mathbf{X}_{2}^{\prime}\right)+\varepsilon\left(\mathbf{X}_{1}-\mathbf{X}_{2}^{\prime}\right) .
$$

When 2 and $2^{\prime}$ are driven by the same signal coming from 1 , the vector fields $\mathbf{X}_{2}(t)$ and $\mathbf{X}_{2}^{\prime}(t)$ in the phase spaces of 2 and $2^{\prime}$ are identical and they can evolve on identical attractors. If initial conditions of both 2 and $2^{\prime}$ lie in the same basin of attraction, then at a certain drive strength, trajectories of both 2 and $2^{\prime}$ will coincide after some transients, i.e., $\left|\mathbf{X}_{2}^{\prime}(t)-\mathbf{X}_{2}(t)\right| \rightarrow 0$ as $t \rightarrow \infty$. In this case the GS between the response system 2 and the drive system 1 builds. On the other hand, a sufficient and necessary condition for GS (or $\mathrm{CS}$ ) is the negativeness of the maximum conditional Lyapunov exponent (MCLE). The conditional Lyapunov exponent spectrum $\left\{\lambda_{T}^{1} \geqslant \lambda_{T}^{2} \geqslant \cdots\right\}$ can be numerically computed along the GS (or CS) manifold. For weak couplings, the maximum exponent $\lambda_{T}^{1}>0$, implying the GS (or CS) manifold is unstable and GS (or CS) between the drive and response is not built. As one increases $\varepsilon, \lambda_{T}^{1}$ becomes negative at certain critical strength, then the evolution of the response element follows the manifold of the drive, i.e.,
$\mathbf{X}_{1}(t)=\mathbf{X}_{2}(t)$ for CS cases and $\mathbf{X}_{2}(t)=\mathbf{G}\left[\mathbf{X}_{1}(t)\right]$ for GS cases. Intermittent deviations from the synchronized manifold can be observed near the synchronization threshold due to the chaotic nature of the attractor.

Now let us consider the mutually coupled case. For $N$ chaotic oscillators with nearest-neighbor couplings, one may write down the following equations of motion:

$$
\dot{\mathbf{X}}_{i}=\mathbf{F}_{i}\left(\mathbf{P}_{i}, \mathbf{X}_{i}\right)+\varepsilon \mathbf{D}\left(\mathbf{X}_{i+1}-\mathbf{X}_{i}\right)-\varepsilon \mathbf{D}\left(\mathbf{X}_{i}-\mathbf{X}_{i-1}\right),
$$

where $\mathbf{D}$ denotes the coupling matrix, and $\varepsilon$ is the coupling strength. First we consider the simplest case $N=2$, where the equations of motion are

$$
\dot{\mathbf{X}}_{1,2}=\mathbf{F}_{1,2}\left(\mathbf{P}_{1,2}, \mathbf{X}_{1,2}\right)+\varepsilon \mathbf{D}\left(\mathbf{X}_{2,1}-\mathbf{X}_{1,2}\right) .
$$

Now we propose the approach in studying the GS behavior between 1 and 2. In fact, the auxiliary-element criterion in studying GS in drive-response cases is still available. Due to the bidirectional interaction, one should introduce two auxiliary systems $1^{\prime}$ and $2^{\prime}$, which, respectively, are identical to 1 and 2, i.e., let 1 drive $2^{\prime}$ and 2 drive $1^{\prime}$, as shown in Fig. 1 (b). The equations of motion of $1^{\prime}$ and $2^{\prime}$ have the same form as 1 and 2, respectively,

$$
\dot{\mathbf{X}}_{1,2}^{\prime}=\mathbf{F}_{1,2}\left(\mathbf{P}_{1,2}, \mathbf{X}_{1,2}^{\prime}\right)+\varepsilon \mathbf{D}\left(\mathbf{X}_{2,1}-\mathbf{X}_{1,2}^{\prime}\right) .
$$

$1^{\prime}$ and $2^{\prime}$ possess the same parameters as 1 and 2, but evolve from different initial conditions. Thus the vector fields $\mathbf{X}_{1,2}(t)$ and $\mathbf{X}_{1,2}^{\prime}(t)$ in the phase spaces of 1 (or 2) and 1' (or $\left.2^{\prime}\right)$ are identical and they can evolve on identical attractors. With increasing the coupling strength, One may expect that both $1^{\prime}-1$ and $2^{\prime}-2$ coincide after the initial transients. Will these two pairs become synchronized at the same minimum coupling strength? Or they achieve synchronization at different coupling thresholds? In other words, will 1 (or 2) be tamed by 2 (or 1 ) for a smaller coupling or 1 and 2 are tamed by each other at the same coupling threshold? To answer this question, let us adopt the $x$-coupled Lorenz oscillators as an example (the coupling is only on the equation of motion of the $x$ component), where the coupling matrix $\mathbf{D}_{11}=\mathbf{1}$ and $\mathbf{D}_{i j}=\mathbf{0}$ for $i \neq 1$ and $j \neq 1$. The equation of motion for a single Lorenz oscillator is written as

$$
\begin{aligned}
& \dot{x}=\sigma(y-x), \\
& \dot{y}=r x-y-x z, \\
& \dot{z}=-b z+x y .
\end{aligned}
$$

Here $\sigma=10, b=8 / 3$, and $r$ is different for different oscillators. For the case $N=2$, we use $r_{1,2}=40,35$, respectively. To measure the degree of synchronization, we introduce the instantaneous distance between the $i$ th oscillator and its auxiliary partner as

$$
\begin{aligned}
D_{i}(t) & =\left\|\mathbf{X}_{i}(t)-\mathbf{X}_{i}^{\prime}(t)\right\| \\
& =\sqrt{\left(x_{i}^{\prime}-x_{i}\right)^{2}+\left(y_{i}^{\prime}-y_{i}\right)^{2}+\left(z_{i}^{\prime}-z_{i}\right)^{2}} .
\end{aligned}
$$



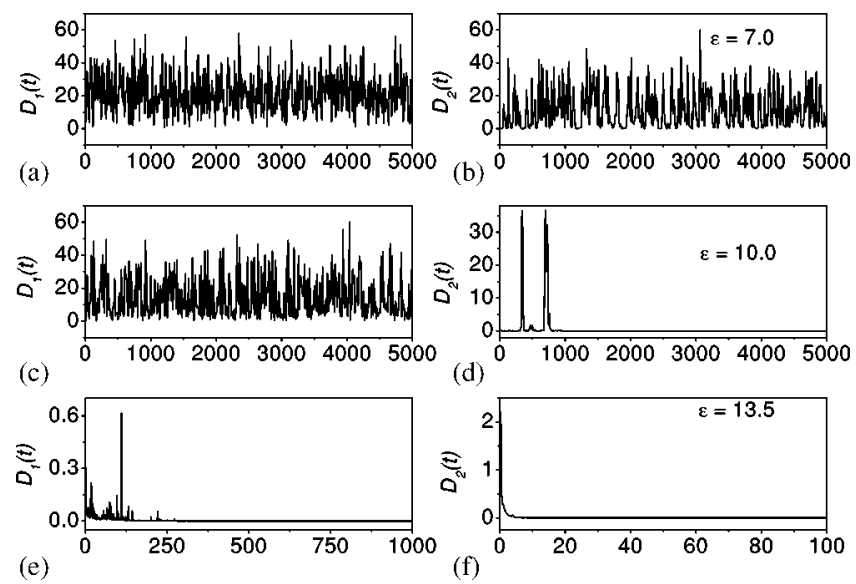

FIG. 2. The evolutions of the difference function $D_{i}(t)$ for different coupling strengths for $N=2$ Lorenz oscillators, where $\varepsilon$ $=7.0$ in (a) and (b), $\varepsilon=10$ in (c) and (d), and $\varepsilon=13.5$ in (e) and (f). Partial generalized synchronization can be observed in (d), and the global generalized synchronization is achieved in (e) and (f).

In Fig. 2, the evolution of the differences $D_{1}(t)$ and $D_{2}(t)$ for two coupled Lorenz oscillators are given at different coupling strengths. In Figs. 2(a) and 2(b), for $\varepsilon=7.0$, it can be found that the differences oscillate irregularly around a nonzero value. This strong fluctuation indicates 1 and 2 are less correlated; With increasing the coupling, one may find a stronger correlation. For example in Figs. 2(c) and 2(d), at $\varepsilon=10$, one of the differences, $D_{2}(t)$, goes to zero, while $D_{1}(t)$ is still in a strongly fluctuating manner. This indicates that under the drive of $1,2^{\prime}$ synchronizes to 2 in the long time limit. This behavior is much similar to that occurs for the drive-response system, when the GS takes place. While in another coupling direction, $1^{\prime}$ does not follow 1 . Therefore for the mutually coupled case, GS is reached first in one of the coupling directions, i.e., 2 is first "slaved" by 1 (the element 1 , as the master, tames the element 2). We call this a partial GS. This result is very interesting because for mutually coupled nonidentical chaotic oscillators, the two coupling directions are not equivalent, that is, synchronizations in the two directions cannot occur at the same minimum threshold. There are always a master oscillator and a slave oscillator. When one further increases the coupling strength, as shown in Figs 2(e) and 2(f) for $\varepsilon=13.5$, one fairly expects that both $D_{1}(t)$ and $D_{2}(t)$ go to zero in the long run. In this case, not only can 1 tame 2, but also 2 tames 1 . They organize themselves to follow each other and enter a global generalized synchronization state.

In fact, the above GS behavior can be well understood by observing the maximum Lyapunov exponent $\lambda_{\max }$ for a single Lorenz oscillator against $r$, as shown in Fig. 3. Because $r$ relates to the Reynolds number in studies of turbulence, $\lambda_{\max }$ monotonically increases with increasing $r$ (except periodic windows). Therefore one has $\lambda_{\max }(r=35)$ $<\lambda_{\max }(r=40)$. The above studies of GS indicate that a less chaotic system can be easier to be entrained. For systems with a larger maximum Lyapunov exponent, higher stochasticity prevents it from being first tamed. Therefore we find that the oscillator with $r=35$ is first synchronized, then the one with $r=40$.

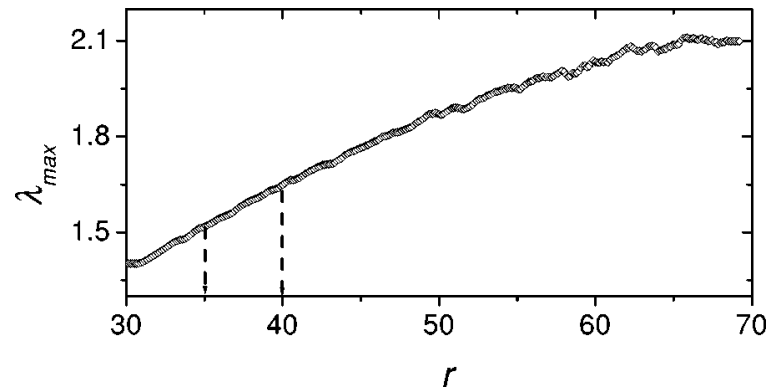

FIG. 3. The maximum Lyapunov exponent of the Lorenz oscillator against $r$.

To better demonstrate this route from partial GS to the global GS for $N=2$ and meanwhile determine the thresholds for GS, we plot the average differences

$$
D_{i}=\lim _{T \rightarrow \infty} \frac{1}{T} \int_{0}^{T} D_{i}(t) d t .
$$

It can be clearly found from Fig. 4(a) that there are two thresholds. At the first threshold $\varepsilon_{c}^{1} \approx 8.4$ one finds $D_{2}=0$, indicating the occurrence of the first GS, where 2 is slaved by 1 . Because in the other direction one still has a nonzero average difference, one only has a partial GS. The second threshold is $\varepsilon_{c}^{2} \approx 13.4$, while $D_{1}$ becomes zero. This is the threshold for a global GS. In Fig. 4(b), one computes the transversal (conditional) Lyapunov exponents $\lambda_{T}$ along the GS manifold. One only needs the maximum exponents $\lambda_{T}^{1}$ and $\lambda_{T}^{2}$ to test the GS in two coupling directions. An excellent agreement with Fig. 4(a) can be found, where at each GS threshold one of the MCLEs becomes negative.

A critical issue is about the robustness of the partial GS and global GS, as well as the basin of attractions of the GS state. As required by the stability of GS, initial states for a partner should lie in the same basin of attraction as the oscillator. For the Lorenz attractor with two foliages, GS can
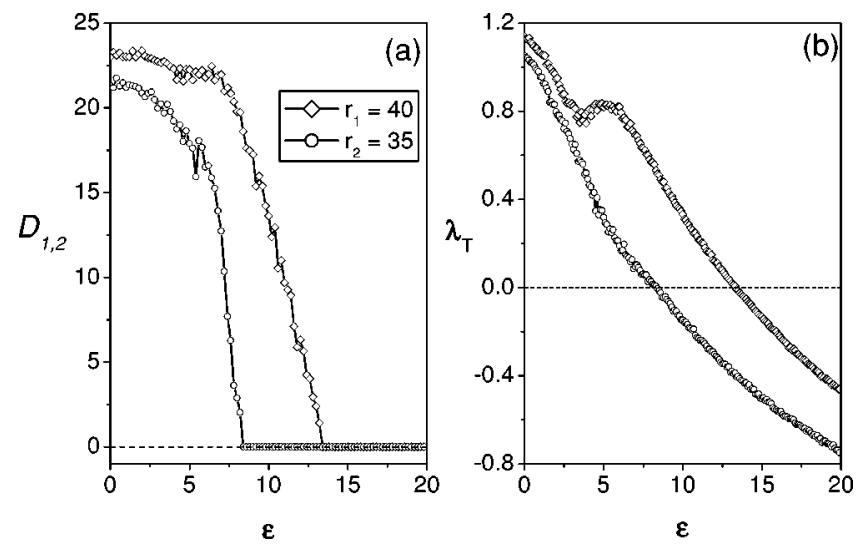

FIG. 4. (a) The averaged distances $D_{i}$ against the coupling strength. They approach zero at different threshold couplings, indicating the achievement of generalized synchronizations. (b) The maximum conditional Lyapunov exponents for the two auxiliary units. Generalized synchronizations are achieved when they pass the zero line. 

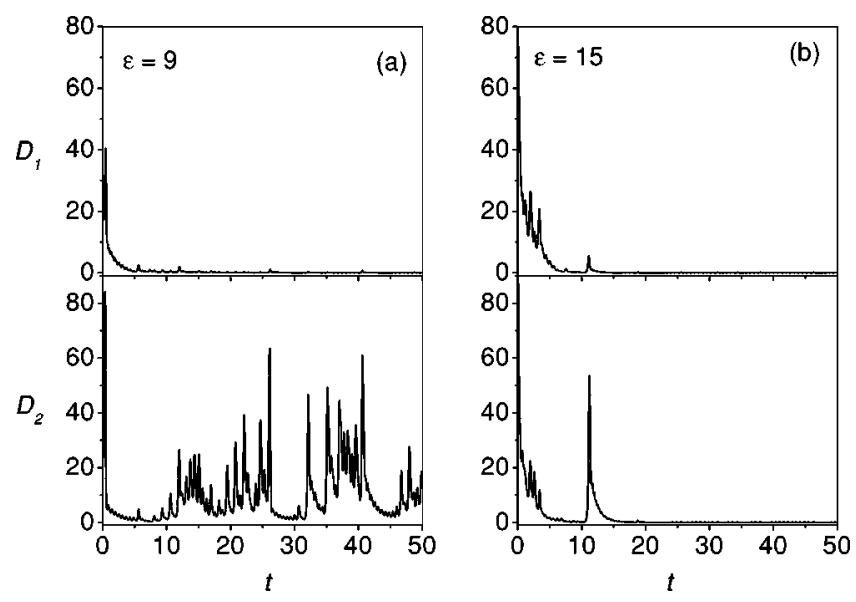

FIG. 5. The evolution of $D_{1,2}(t)$ with the same parameters as in Fig. 2 for $\varepsilon=9.0$ and 15.0, where the auxiliary oscillator evolves from a $\left\{-\mathbf{X}_{i}(0)+\delta_{i}\right\}$ with $\delta_{i}$ being small random numbers.

still be attained even if the oscillator and its partner evolve from completely different foliages due to the ergodicity of the attractor. In Fig. 5(a) and 5(b), we give the evolution of $D_{1,2}(t)$ with the same parameters as in Fig. 2 for $\varepsilon=9.0$ and 15.0, where the auxiliary oscillator evolves from an initial condition $\mathbf{X}_{i}^{\prime}(0)=-\mathbf{X}_{i}(0)+\delta_{i}$ with $\delta_{i}$ being small random numbers. It can be clearly found that for $\varepsilon=9.0, D_{1} \rightarrow 0$, i.e., a partial GS can still be achieved. For $\varepsilon=15$, both $D_{1}$ and $\mathrm{D}_{2}$ approach 0 , indicating a global GS. Here we did not exclude the possibility of a failure of GS for other coupled systems or other forms of coupling. One can find examples that GS is not satisfied $[19,20]$. But for the present system we did not find this violation. The scenario of transitions from partial GS to global GS proposed in this paper is typical (not universal), which can be observed in many systems. To further illuminate it, we may test a typical case of coupled Lorenz-Rossler systems, where the Lorenz and Rossler possess rather different types of chaotic motions. For the $x$-coupled case one has

$$
\begin{gathered}
\dot{x}_{1}=\sigma\left(y_{1}-x_{1}\right)+\varepsilon\left(x_{2}-x_{1}\right), \\
\dot{y}_{1}=r x_{1}-y_{1}-x_{1} z_{1}, \\
\dot{z}_{1}=-b z_{1}+x_{1} y_{1}, \\
\dot{x}_{2}=-\omega x_{2}-z_{2}+\varepsilon\left(x_{1}-x_{2}\right), \\
\dot{y}_{2}=\omega x_{2}+a y_{2}, \\
\dot{z}_{2}=f+z_{2}\left(x_{2}-c\right),
\end{gathered}
$$

where for Lorenz $\sigma=10, b=8 / 3, r=35$, and for Rossler $a$ $=0.165, \omega=5.5, f=0.2, c=10$. We test the GS dynamics by using the same way as above. In Fig. 6(a), we computed the average differences $D_{1,2}$ against the coupling. At a very weak coupling $\varepsilon_{c}^{1} \approx 1.2, D_{2} \rightarrow 0$, indicating that the Rossler oscillator is easier to be entrained. Due to the stronger stochasticity of the Lorenz oscillator, it is more difficult to get
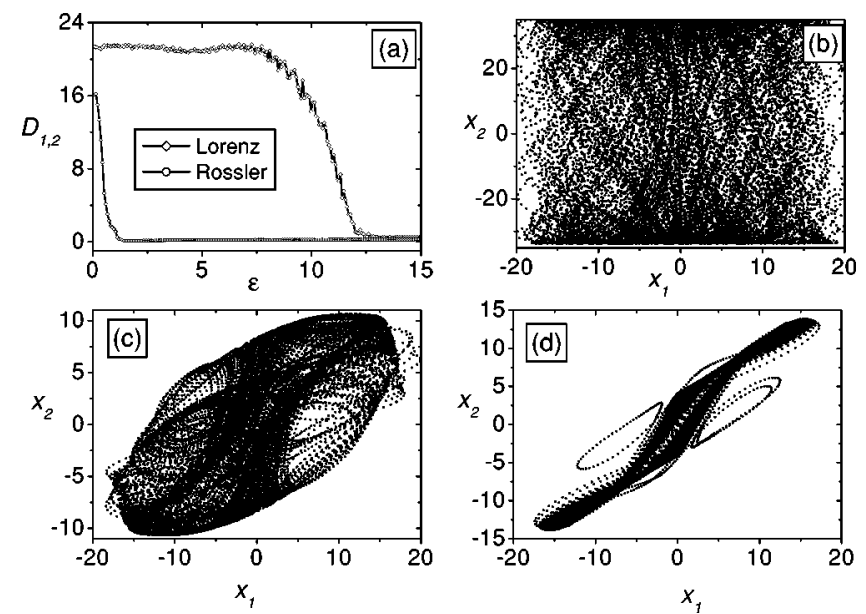

FIG. 6. (a): The average differences $D_{1,2}$ against the coupling. The Rossler oscillator is easier to be entrained. (b) -(d): The relation between $x_{2}$ and $x_{1}$ for $\varepsilon=0.5$ (unsynchronized state), $\varepsilon=5.0$ (partially synchronized state), and $\varepsilon=13.0$ (global GS state).

synchronized. We find the threshold is at $\varepsilon_{c}^{2} \approx 12.3$. We also give the relation between $x_{2}$ and $x_{1}$ for different couplings. In Fig. 5(b), for $\varepsilon=0.5$ (unsynchronized state), it is shown that $x_{2}$ and $x_{1}$ are almost uncorrelated. For $\varepsilon=5.0$ (partially synchronized state), $x_{2}$ and $x_{1}$ has certain degree of correlations, as shown in Fig. 6(c). In the global GS regime, e.g., at $\varepsilon=13.0, x_{2}$ and $x_{1}$ fall onto a simpler manifold, as shown in Fig. 6(d) for darker regions. Deviations from the global GS manifold can also be observed due to the typical intermittency near the synchronization threshold.

Now it is important to extend the present idea to groups of $N>2$ coupled oscillators in order to verify the validity of the above scenario. Taking the $N=3$ case as an example. Because the three oscillators are coupled together, then for each oscillator, e.g., oscillator 1 , one cannot introduce auxiliary systems $2^{\prime}$ and $3^{\prime}$ that are independent. In this case, one should introduce three auxiliaries, as shown in Fig. 7(a). Each auxiliary oscillator is driven by the other two oscillators (e.g., $1^{\prime}$ is driven by 2 and 3). The equations of motion for the three auxiliary systems have the same form as their masters in the original system. Therefore, one may test which oscillator is the first that can be tamed by others, then the second, and so on. In Fig. 7(b), we give the average differences $D_{i}$ vs the coupling strength $\varepsilon$. Here $r_{1}=50$, $r_{2}=55$, and $r_{3}=60$. It shows a scenario similar to the case of $N=2$. At $\varepsilon_{c}^{1}=6.4, D_{1}=0$, and the oscillator 1 is first synchronized. While in this regime one has $D_{2} \neq 0$ and $D_{3} \neq 0$, thus the oscillators 2 and 3 cannot be tamed by other oscillators in the group. With increasing the coupling, at $\varepsilon_{c}^{2}$ $=8.2$, the oscillator 2 is also slaved; Finally at $\varepsilon_{c}^{3}=10.6$ all average differences become zero, indicating that all oscillators are synchronized. The MCLEs plotted in Fig. 7(c) give a clear picture for the above cascade. Therefore for the $N=3$ case, one also observes a route from partial GS to global GS.

One expects this route is a generic scenario for GS in many coupled chaotic oscillators. For the $N=3$ case, oscillators are coupled with the nearest-neighbor $(\mathrm{NN})$ manner. It is necessary to test the above scenario for a non-NN case. 


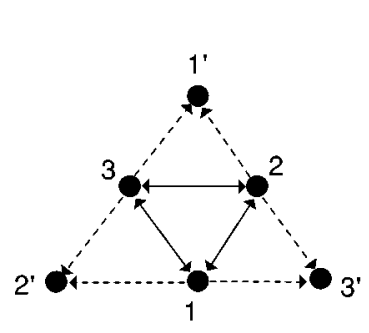

(a)

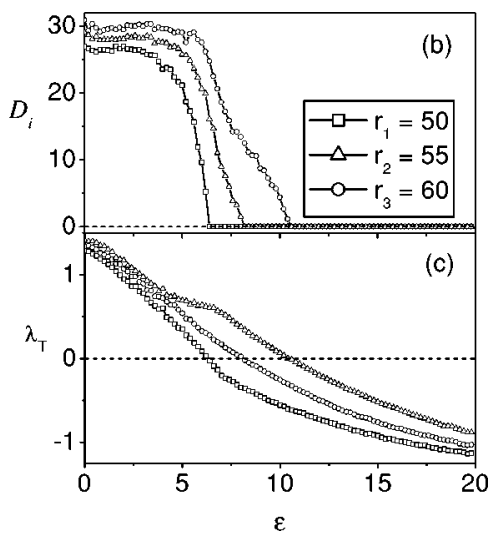

FIG. 7. (a) A schematic plot of the auxiliary approach for $N$ $=3$ mutually coupled oscillators. (b) The average distances vary with the coupling strength. (c) The maximum conditional Lyapunov exponents for the three partners vary against the coupling strength.

Thus we further investigate the case $N=4$, which is a minimal number for non-NN cases. The sketch for the test of GS is shown in Fig. 8(a). The auxiliary elements have the same form of equations of motion: $\dot{\mathbf{X}}_{i}^{\prime}=\mathbf{F}_{i}\left(\mathbf{P}_{i}, \mathbf{X}_{i}^{\prime}\right)+\varepsilon \mathbf{D}\left(\mathbf{X}_{i+1}\right.$ $\left.-\mathbf{X}_{i}^{\prime}\right)-\varepsilon \mathbf{D}\left(\mathbf{X}_{i}^{\prime}-\mathbf{X}_{i-1}\right)$. In Fig. 8(b), the differences between the oscillators and their auxiliary partners are computed against the coupling strength for four Lorenz oscillators with $r_{1,2,3,4}=60,61,62$, and 63 , respectively. One finds a good series for $D_{i}$ becoming zero at different threshold couplings. The MCLEs plotting in Fig. 8(c) also present a clear picture for the cascade of transitions from a partial GS to a global GS. Based on the above studies, one can expect a route from partial GS to global GS for a general $N$ coupled nonidentical oscillators. The present study reveals an intrinsic order embedded in complicated dynamics.

To conclude, in this paper we present the approach to GS in mutually coupled nonidentical chaotic oscillators and a generic route from partial GS to the global GS is proposed. The approach presented in this paper should be also valid for general systems with local or nonlocal, unidirectional or bidirectional couplings, and the scenario from the partial GS to the global GS also should be a generic feature. As we know, the scenario of transitions from a partial order the global

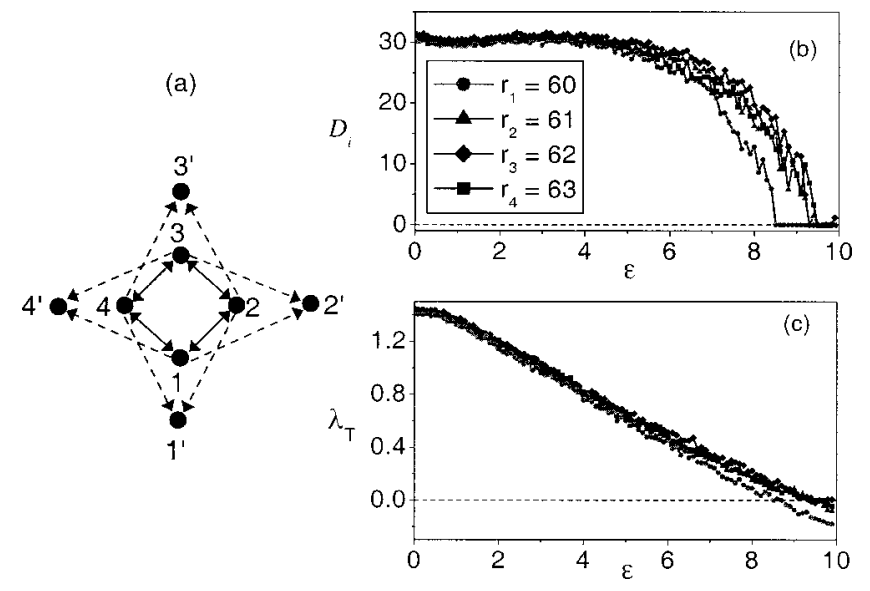

FIG. 8. (a) The auxiliary approach to $N=4$ mutually coupled chaotic oscillators. (b) The same as Fig. 4(b) with $N=4$. (c) The same as Fig. 4(c) with $N=4$.

order has been proposed in other forms of synchronizations, e.g., complete synchronization, phase synchronization, and lag synchronization. We have also found the similar cascade for measuring synchronization for coupled symplectic maps [21]. Therefore we have different kinds of synchronizations for coupled chaotic oscillators, and for each kind of synchronization there exists a cascade of transitions from a local clustering to a global clustering. All these synchronizations reveal different degrees of internal self-organized orders. On the other hand, studies of the boundaries among these different synchronizations and their relations is a subject that needs a long-term exploration [22]. Furthermore, the roles played by synchronizations in spatiotemporal pattern dynamics are also open problems.

Stimulating discussions with Prof. Gang Hu, Dr. Daihai He, and Dr. Meng Zhan are gratefully acknowledged. This work is supported by the National Natural Science Foundation of China, the Special Funds for Major State Basic Research Projects, the Foundation for University key teacher by the Ministry of Education, Special Funds for Excellent Doctoral Dissertations, the TRAPOYT in Higher Education Institutions of MOE, and the Huo-Ying-Dong Educational Funds for Excellent Young Teachers.
[1] L. Fabiny, P. Colet, and R. Roy, Phys. Rev. A 47, 4287 (1993); R. Roy and K.S. Thornburg, Jr., Phys. Rev. Lett. 72, 2009 (1994).

[2] V.S. Anischenko et al., Int. J. Bifurcation Chaos Appl. Sci. Eng. 2, 633 (1992); J.F. Heagy, T.L. Carroll, and L.M. Pecora, Phys. Rev. E 50, 1874 (1994).

[3] I. Schreiber and M. Marek, Physica D 50, 258 (1982); S.K. Han, C. Kurrer, and K. Kuramoto, Phys. Rev. Lett. 75, 3190 (1995).

[4] L. Kocarev and U. Parlitz, Phys. Rev. Lett. 74, 5028 (1983).

[5] Y. Kuramoto, Chemical Oscillations, Waves and Turbulence (Springer-Verlag, Berlin, 1984).

[6] L.M. Pecora and T.L. Carroll, Phys. Rev. Lett. 64, 821 (1990).
[7] N.F. Rulkov et al., Phys. Rev. E 51, 980 (1995); H.D.I. Abarbanel et al., ibid. 53, 4528 (1996); L. Pecora and U. Parlitz, Phys. Rev. Lett. 76, 1816 (1996); K. Pyragas, Phys. Rev. E 54, R4508 (1996).

[8] M.G. Rosenblum, A.S. Pikovsky, and J. Kurths, Phys. Rev. Lett. 76, 1804 (1996); E. Rosa, E. Ott, and M.H. Hess, ibid. 80, 1642 (1998); M.A. Zaks, E. Park, M.G. Rosenblum, and J. Kurths, ibid. 82, 4228 (1999).

[9] M.G. Rosenblum, A.S. Pikovsky and J. Kurths, Phys. Rev. Lett. 78, 4193 (1997); S. Taherion and Y.C. Lai, Phys. Rev. E 59, R6247 (1999).

[10] A. Hampton and D.H. Zanette, Phys. Rev. Lett. 83, 2179 (1999). 
[11] G. Hu, Y. Zhang, H.A. Cerdeira, and S. Chen, Phys. Rev. Lett. 85, 3377 (2000).

[12] G. Hu, J. Yang, and W. Ma, Phys. Rev. Lett. 81, 5314 (1998).

[13] M.A. Matias et al., Phys. Rev. Lett. 78, 219 (1997).

[14] S.H. Strogatz et al., Physica D 31, 143 (1988); J. Phys. A 21, L699 (1988); Phys. Rev. Lett. 68, 2730 (1992); M.K.S. Young and S.H. Strogatz, ibid. 82, 648 (1999).

[15] Z. Zheng, G. Hu, and B. Hu, Phys. Rev. Lett. 81, 5318 (1998); Z. Zheng, B. Hu, and G. Hu, Phys. Rev. E 62, 402 (2000).

[16] G.V. Osipov, A.S. Pikovsky, M.G. Rosenblum, and J. Kurths,
Phys. Rev. E 55, 2353 (1997).

[17] B. Hu and Z. Zheng, Int. J. Bifurcation Chaos Appl. Sci. Eng. 10, 2399 (2000).

[18] U. Parlitz et al., Phys. Rev. E 54, 2115 (1996).

[19] M. de Sousa Vieira, A.J. Lichtenberg, and M.A. Lieberman, Phys. Rev. A 46, R7359 (1992).

[20] M. de Sousa Vieira and A.J. Lichtenberg, Phys. Rev. E 56, R3741 (1997).

[21] Z. Zheng (unpublished).

[22] Z. Zheng and G. Hu, Phys. Rev. E 62, 7882 (2000). 